\title{
On the problem of optimal timing of slaughtering in fish farming
}

\section{J. LILLESTØL†}

Keywords: Optimization, stochastic systems, control theory, optimal stopping rules.

Various issues concerning optimal feeding and optimal slaughtering are studied, following different formulations of the problem. We discuss separate and joint modeling, 'one-step look-ahead' and global procedures, deterministic and stochastic models. The price functions involved typically have jumps going from one weight category to the next. Special emphasis is given to two models, one with simplifying assumptions on the weight distribution, the other with a linear price approximation.

\section{Introduction}

The optimal management of a fish farm may be looked upon as the choice of a combined policy of (i) gathering information, (ii) feeding, and (iii) timing the slaughtering. The problem is of a sequential nature, at each stage projections of fish weights at future points in time are needed. The projections will depend on the sampling done, and the (sometimes limited) knowledge of the relationship between feeding and growth. Growth is also affected by outside factors, the main factor being sea temperature. This dependence is highly non-linear, in the sense that the intake of feed is low at both low and high temperatures. The combined policy is affected by various cost factors: feed costs, feeding costs, sampling costs and interest rates, and is also affected by the (uncertain) market price of the fish.

The main challenge for the decision analyst is to provide models, and estimates for parameters therein, which may be put to work in a prospective decision-making context. This means that explanatory growth models with many variables and even good explanatory power (in retrospect), must be foregone for the use of simpler models, preferably with some adaptive features and possibility of user interaction. An extensive literature exists, for growth curves in general, and for refined models involving the bioenergetics. However such models are not necessarily suitable for decision-making purposes, and a fresh look is needed. In this paper we focus on (ii) feeding and (iii) timing of slaughtering, with main emphasis on the latter. Some details which are left out here may be found in Lillestøl (1984), where also questions concerning (i) the gathering of information are discussed, and in Lillestøl (1985) where control-theoretic aspects are in focus.

Our investigations are mainly concerned with farming the Atlantic salmon (salmo salar) in enclosures with typical population sizes of 5000 and upwards. Since

Received 15 January 1987.

This paper was presented at the IFAC Symposium on Automation and Dataprocessing in Aquaculture, Trondheim, Norway, 18-20 August 1986.

This paper is reprinted with the permission of IFAC.

+ Center for Applied Research, The Norwegian School of Economics and Business Administration, Helleveien 30, 5035 Bergen-Sandviken, Norway. 
the market price of the fish will typically differ for different weight categories, a proper decision model will have to account for the distribution of weights at any instant when slaughtering is considered. We have analysed data from five enclosures provided by the Aquaculture Research Station at Austevoll, Norway. Extensive weighings at ten different times revealed weight distributions close to the normal, with a nearly constant coefficient of variation (standard deviation divided by mean) of about $\mathbf{0 \cdot 2 0}$. This may be utilized in decision models, and free the decision maker (i.e. the fish farmer) from thinking about standard deviations. However, the coefficient of variation may, to some extent, vary with heredity, sorting of the smolt, environment and feeding procedures. In another experiment, the coefficient of variation was again fairly constant over time, but at a higher level, close to $0 \cdot 30$, a possible reason being less well sorted smolt at the outset.

The problems of optimal feeding and optimal slaughtering can be studied, following different formulations. We have choices between separate and joint modeling, 'one-step look-ahead' and global procedures, deterministic and stochastic models. The most ambitious formulation is joint, global, stochastic models requiring optimal control theory. However we have found it fairly difficult to model the dynamics of such models properly, even in the deterministic case. Moreover they may be hard to implement in practice, since the solutions are heavily dependent on practice, since the solutions are heavily dependent on precise parameter estimates. Consequently we prefer (possibly suboptimal) 'one-step look-ahead' procedures to global optimization models. Simple models of this type should be preferred to the sophisticated ones, both for statistical reasons and user-friendliness. Models with many variables may lead to worse predictions, because of the estimation of many parameters which may change over time.

\section{Optimal slaughtering}

Consider a population of fish in an enclosure, where $\mu_{t}$ is their average weight at time $t$, and $\sigma_{t}$ is the corresponding standard deviation. The weight $X_{t}$ of a random fish at time $t$ is taken as a random variable with probability distribution expressed by a density $f(x ; t)$. We may then write

$$
\begin{aligned}
& \mu_{t}=E X_{t}=\int_{-\infty}^{\infty} x f(x ; t) d x \\
& \sigma_{t}^{2}=\operatorname{var} X_{t}=\int_{-\infty}^{\infty}\left(x-\mu_{t}\right)^{2} f(x ; t) d x
\end{aligned}
$$

Let $p(x ; t)$ be the price per kilo for fish of weight $x$ at time $t$. The expected pay for a random fish at time $t$ will then be

$$
V(t)=\int_{-\infty}^{\infty} p(x ; t) x f(x ; t) d x
$$

Consider the problem of deciding whether to slaughter (all) the fish now at time $t$, or postpone slaughtering till time $t+h$, where expected pay is $V(t+h)$. In the latter case we incur extra feeding costs $R_{t} . C_{t} . h$. Here $R_{t}$ is a feed-rate at time $t$, defined as the fed amount (in kilo) per unit of time per fish and $C_{t}$ is the price per kilo feed at time $t$. The decision whether to slaughter at time $t$ or postpone it to time $t+h$ may be judged from the inequalities

$$
V(t+h)-R_{t} C_{t} h \gtrless\left(1+\rho_{t} h\right) V(t)
$$


where $\rho_{t}$ is a (continuous) discount factor which may both reflect current interest rates and various risk factors at time $t$ (death, escape, maturity etc.). Reshuffling terms, division by $h$ and letting $h$ tend to zero gives

$$
V^{\prime}(t)-R_{t} . C_{t}-\rho_{t} V(t) \gtrless 0
$$

The decision rule is then to postpone slaughtering as long as the expression stays positive, but slaughter as soon as the expression becomes negative. This rule is derived by one-step (or infinitesimal) look-ahead considerations. It is well known that myopic rules are not generally globally optimal. It may happen that a negative sign occurs at an early stage because of temporarily slow weight gain compared to feeding and capital costs. However if the rule is followed only after slaughtering is regarded as a possible action, this is not likely to happen for a proper specification of the parameters involved.

In order to use formula (2) we have to compute $V(t)$ and its derivative $V^{\prime}(t)$ from (1) under reasonable assumptions on the price function $p(x ; t)$ and the weight distribution $f(x ; t)$. We will provide two solutions here, one which makes a restrictive assumption on $p$ but not on $f$ and another which makes a restrictive assumption on $f$, and not on $p$.

The price function $p(x ; t)$ is, for each $t$, an increasing step function in $x$, i.e. constant over weight intervals. Our analysis of actual price data shows that these step functions are reasonably well approximated by linear functions of $x$, that is

$$
p(x ; t)=a(t)+b(t) \cdot x
$$

for suitably chosen $a(t)$ and $b(t)$. With this assumption we get the expected price of a random fish as

$$
\begin{aligned}
V(t) & =\int_{-\infty}^{\infty} p(x ; t) x f(x ; t) d x \\
& =a(t) \int_{-\infty}^{\infty} x f(x ; t) d x+b(t) \int_{-\infty}^{\infty} x^{2} f(x ; t) d x \\
& =a(t) \cdot \mu_{t}+b(t) \cdot\left(\sigma_{t}^{2}+\mu_{t}^{2}\right) \\
& =a(t) \cdot \mu_{t}+b(t) \mu_{t}^{2}\left(1+\kappa_{t}^{2}\right)
\end{aligned}
$$

where $\kappa_{t}=\sigma_{t} / \mu_{t}$ is the coefficient of variation at time $t$. This result is not dependent on any assumptions on the weight distribution, say of normality. The distribution may even be skew, but the skewness does not affect the expected price. If we had used a quadratic assumption

we get instead

$$
p(x ; t)=a(t)+b(t) \cdot x+c(t) \cdot x^{2}
$$

$$
V(t)=a(t) \cdot \mu_{t}+b(t) \cdot \mu_{t}^{2}\left(1+\kappa_{t}^{2}\right)+c(t) \cdot \mu_{t}^{3}\left(1+3 \kappa_{t}^{2}+\kappa_{t}^{3} \cdot \lambda_{t}\right)
$$

where $\lambda_{t}$ is the usual measure of skewness of a distribution, i.e. the third central moment divided by $\sigma_{t}^{3}$. For symmetric distributions (among them the normal) $\lambda_{t}=$ 0 , and the expected price depends only on $\mu_{t}$ and $\kappa_{t}$ (or $\sigma_{t}$ ).

Assuming that a linear price function is sufficient, we will now look at the computation of $V^{\prime}(t)$. The general expression is fairly complicated, and some simplifying assumptions may be needed. In the case of zero price-time derivative and constant coefficient of variation $\kappa_{t}=k$, we get

$$
V^{\prime}(t)=a(t) \cdot \mu_{t}^{\prime}+b(t) \cdot 2\left(1+k^{2}\right) \mu_{t} \cdot \mu_{t}^{\prime}
$$


In order to exercise the rule (2), we have to specify the feed-rate $R_{t}$, the weight $\mu_{t}$ and its derivative $\mu_{t}^{\prime}$ in addition to the economic parameters. Fish farmers often express themselves in terms of feeding per kilo biomass and feed-factors. This may be formalized by the identities

$$
\begin{aligned}
& R_{t}=r_{t} \cdot \mu_{t} \\
& R_{t}=\phi_{t} \cdot \mu_{t}^{\prime}
\end{aligned}
$$

Here $r_{t}$ is the fed amount (in kilo) per unit of time, per kilo fish at time $t$, and $\phi_{t}$ is the (local) feed-factor at time $t$, i.e. the amount of feed required to produce a one unit weight increase. One possibility is to let $\phi_{t}$ and $r_{t}$ determine the parameters in (2). As an approximation they may be taken as constant over time for the given feed, possibly corrected by specific information available at time $t$ (e.g. temperature) or the history up to time $t$ (e.g. diseases and medication). With an estimate of $\mu_{t}$ we now obtain $R_{t}$ by (3) and then $\mu_{t}^{\prime}$ from (4).

Some additional comments on parameter specifications are the costs of slaughtering a fish $S(t)$ may be taken into account by replacing $V(t)$ by $V(t)-S(t)$ and $V^{\prime}(t)$ by $V^{\prime}(t)-S^{\prime}(t)$ in (2). In the common case of constant $S(t)=S$ when $S^{\prime}(t)=0$, this may also be taken into account by downgrading the price function $p$. Typically interest rates are constant so that $\rho_{t}=\rho$. However if we want to include the risk of loss by escape or death in the analysis, we may replace $\rho_{t}$ by $\rho_{t}^{+}=\rho_{t}+\delta_{t}$ where $\delta_{t}$ is the loss-rate at time $t$. The risk of maturity may also be included here.

\section{Example}

The Fish Farmers Sales Organization in Norway offered on 07.07 .83 the following prices for a certain quality

$\begin{array}{lccccc}\text { Weight (in kilo) } & <2 & 2-3 & 3-4 & 4-5 & 5-6 \\ \text { Price in N.kr. } & - & 30 \cdot 50 & 34 \cdot 50 & 38 \cdot 50 & 42 \cdot 50 \\ \text { Weight (in kilo) } & 6-7 & 7-8 & 8-9 & >9 & \\ \text { Price in N. kr. } & 46 \cdot 50 & 50 \cdot 50 & 54 \cdot 50 & - & \end{array}$

If we identify the prices in each weight group by the midpoint in the interval, we get exactly the relationship

$$
p=20 \cdot 5+4 \cdot 0 . x
$$

Let us assume that currently $\mu_{t}=4.0$ and $\kappa_{t}=0.2$ while $\mu_{t}^{\prime}=0.025$ with week as time unit, for example using a feed-rate of 0.1 kilo feed per week per fish, and assuming a feed-factor of 4.0 . We then get

$$
\begin{aligned}
V(t) & =20.5 .4 \times 0+4.0 \times 4.0^{2}\left(1+0.2^{2}\right)=148.6 \quad(148 \cdot 56) \\
V^{\prime}(t) & =20.5 \times 0.025+4.0 \times 2 \times\left(1+0.2^{2}\right) \times 4.0 \times 0.025=1.342
\end{aligned}
$$

Here the numbers in parenthesis are found by the alternative theory below. Using a constant discount rate of 20 per cent per year we get $\rho=0.003506$, and for $C_{t}=8.2$ the left hand side of (2) becomes zero. If the current price of feed is greater than N.kr. 8.20 it will be profitable to slaughter at once.

Let us take an alternative approach with exact prices, but with simplifying distributional assumptions: Define $l+1$ weight groups by separating points $a_{1}, a_{2}$, $\ldots, a_{l}$ so that weight group no. $\mathrm{i}$ is given by the interval $\left(a_{i}, a_{i+1}\right)$ for $i=0,1,2, \ldots, l$ 
(set $a_{0}=-\infty$ and $a_{l+1}=+\infty$ ). The fraction of fish in group no. $\mathrm{i}$ at time $t$ is

$$
q_{i}(t)=\int_{a_{i}}^{a_{i+1}} f(x ; t) d x \quad i=0,1,2, \ldots, l
$$

Suppose that the price per kilo in group no. i at time $t$ is $p_{i}(t)$. The expected price of a random fish now becomes

$$
V(t)=\sum_{i=0}^{l} p_{i}(t) \int_{a_{i}}^{a_{i+1}} x f(x ; t) d x=\sum_{i=0}^{l} p_{i}(t) m_{i}(t)
$$

where we have introduced

$$
m_{i}(t)=\int_{a_{t}}^{a_{i+1}} x f(x ; t) d x
$$

which is the contribution to the average weight from the fish in group no. i. (Note that $m_{0}(t)+m_{1}(t)+\cdots+m_{l}(t)=\mu_{t}$.) It follows that

$$
V^{\prime}(t)=\sum_{i=0}^{l} p_{i}(t) m_{i}^{\prime}(t)+\sum_{i=0}^{l} p_{i}^{\prime}(t) m_{i}(t)
$$

In the case of a normal weight distribution we have

$$
f(x ; t)=f\left(x ; \mu_{t}, \sigma_{t}\right)=\frac{1}{\sigma_{t}} g\left(\frac{x-\mu_{t}}{\sigma_{t}}\right)
$$

where $g$ is the standard-normal density. In this case

$$
q_{i}(t)=\int_{a_{i}}^{a_{i+1}} f\left(x ; \mu_{t}, \sigma_{t}\right) d x=G\left(\frac{a_{i+1}-\mu_{t}}{\sigma_{t}}\right)-G\left(\frac{a_{i}-\mu_{t}}{\sigma_{t}}\right)
$$

where $G$ is the cumulative standard-normal distribution. Introduce the functions

$$
H_{i}^{j}(t)=a_{i+1}^{j} \cdot f\left(a_{i+1}^{j} ; t\right)-a_{i} \cdot f\left(a_{i} ; t\right) \text { for } j=0,1,2
$$

By partial integration in the defining formula we get

$$
m_{i}(t)=\mu_{i}\left(q_{i}(t)-\frac{\sigma_{t}^{2}}{\mu_{t}} H_{i}^{0}(t)\right)
$$

We need expressions for the derivatives. Neglecting higher order terms we have

$$
q_{i}^{\prime}(t) \approx \int_{a_{i}}^{a_{i+1}}\left(\frac{\partial f}{\partial \mu_{t}} \cdot \frac{d \mu_{t}}{d t}+\frac{\partial f}{\partial \sigma_{t}} \cdot \frac{d \sigma_{t}}{d t}\right) d x
$$

It is easy to check that

$$
\frac{\partial f}{\partial \mu}=\frac{x-\mu}{\sigma} \cdot \frac{1}{\sigma} \cdot f \quad \frac{\partial f}{\partial \sigma}=\left(\left(\frac{x-\mu}{\sigma}\right)^{2}-1\right) \cdot \frac{1}{\sigma} \cdot f
$$

If we assume that $\kappa_{t}=\sigma_{t} / \mu_{t}=$ constant, we get the following expressions

$$
\begin{aligned}
q^{\prime}(t) & =-\frac{\mu_{t}^{\prime}}{\mu_{t}} H_{i}^{1}(t) \\
m_{i}^{\prime}(t) & =\frac{\mu_{t}^{\prime}}{\mu_{t}}\left(m_{i}(t)-H_{i}^{2}(t)\right)
\end{aligned}
$$


Although these expressions look unpleasant, they are easy to program, and require only standard-normal density and cumulative probability evaluations. In the example above, we found that the two routes of approximate reasoning led to almost identical results.

\section{Global modeling}

In this section we discuss some of the problems encountered by global optimization. The problem of optimal timing of slaughtering is to determine

$$
\max _{0 \leqslant t \leqslant T}\left\{V(t) \exp \left(-\rho_{t} t\right)-\int_{0}^{t} R_{u} C_{u} \exp \left(-\rho_{u}, u\right) d u\right\}
$$

where $T$ is an upper time limit for keeping the fish. If $V(t)$ is a differentiable function of $t$, a necessary condition for an external point is

$$
V^{\prime}(t)-R_{t} C_{t}-\left(\rho_{t}+t \rho_{t}^{\prime}\right) V(t)=0
$$

which may occur for several $t$ s corresponding local extrema. This is identical to the left hand side of (2) in two cases, for $\rho_{t}=\rho$ (constant) and for $t=0$ regardless of $\rho_{0}$. In the case that we have kept the fish till time $t_{0}$ without slaughtering, we are tempted to take this as a new origin, i.e. take $t=0$ in a renewed global optimization. The new optimal $t$ will then be pushed slightly ahead of the true optimal $t$. If we want to take into account the risk of loss by death and escape we have to replace $\rho_{t}$ by

$$
\rho_{t}^{+}=\rho_{t}+\frac{1}{t} \int_{0}^{t} \delta_{u} d u
$$

where $\delta_{u}$ is the loss-rate at time $u$. In this case $\rho_{t}+t \rho_{t}^{\prime}$ have to be replaced by $\delta_{t}+\rho_{t}+t \rho_{t}^{\prime}$ in the condition (5).

In order to compute $V(t)$ in (5) we may specify

(i) $R_{t}$ and $\mu_{t}$ independently

(ii) $R_{t}$ as a response to attained weight $\mu_{t}$

(iii) $\mu_{t}^{\prime}$ as a response to the feed-rate $R_{t}$

(iv) $R_{t}$ and $\mu_{t}$ consistent with some feed-back model.

Alternative (i) is the simplest, but may be claimed to be too naïve. For (ii) we make assumptions on $r_{t}$ and use (3), where the result should be consistent with expected feed-factors $\phi_{t}$ given by (4). For (iii) we make assumptions on $\phi_{t}$ and use (4), where the result should be consistent with expected weight and formula (3), which can alternatively be written

$$
\mu_{t}=\mu_{0}+\int_{0}^{t}\left(R_{u} / \phi_{u}\right) d u=\mu_{0} \cdot \exp \left\{\int_{0}^{t}\left(r_{u} / \phi_{u}\right) d u\right\}
$$

Of course (iv) is the most realistic case, but may be difficult to model. The choice of specification will be a compromise between our ambitions concerning realism, and simplicity and ease of implementation. It is clear that the possibilities of obtaining reliable estimates of the unknowns should affect our choice of specification. Moreover the interpretation of available data may differ according to our choice among (i)-(iv). Let us consider a simple example of type (i). 


\section{Example}

Consider year as time unit, and the weight function

$$
\mu_{t}=4 \cdot 5 t^{2}-1 \cdot 5 t^{3}
$$

displayed below. We see that it is increasing up to $t=2$, and decreasing afterwards. It may seem odd to use a weight function with this feature, but we feel no need to have good approximation to real growth in the region where slaughtering is unfeasible anyway, i.e. after extensive maturation.

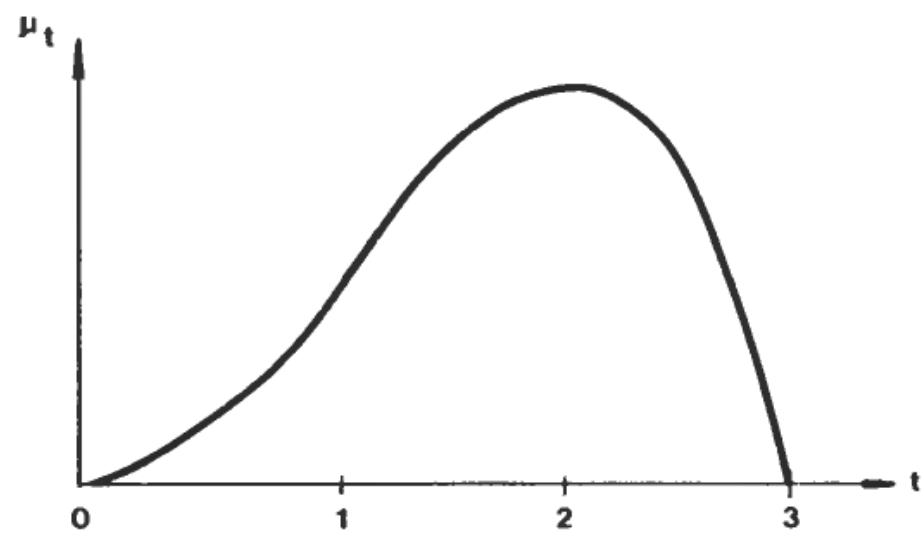

Figure 1. A third degree growth function.

Assume a constant feed-rate per kilo fish, of $r_{t}=2.0 \mathrm{~kg}$ (per year), so that $R_{t}=$ $2 \mu_{t}$. Furthermore assume a constant coefficient of variation $\kappa_{t}=0 \cdot 2$, and the approximate price function of the previous example. The optimal time of slaughtering for some combinations of feed prices and interest rates are given in table 1 (number of months in parenthesis).

\begin{tabular}{cccc}
\hline & \multicolumn{3}{c}{ Interest rate in per cent per year } \\
\cline { 2 - 4 } Feed price \\
per kilo & 15 & 20 & 25 \\
\hline 5 & $1.832(22.0)$ & $1.812(21 \cdot 7)$ & $1.790(21.5)$ \\
10 & $1.716(20 \cdot 6)$ & $1.692(20.3)$ & $1.670(20.0)$ \\
15 & $1.586(19.0)$ & $1.560(18.7)$ & $1.536(18.4)$ \\
\hline
\end{tabular}

Table 1. The optimal time of slaughtering.

We claim no realism in the particular specification given, but third degree polynomials pick up essential features of actual growth, and are easily estimated from real data.

\section{Joint global modeling}

In this final section we give a brief survey of our efforts to develop global models for the joint optimization of feeding and timing of slaughtering. Only deterministic models are mentioned, but the general comments affect stochastic models as well. 
Let $x(t)$ be the weight of the fish at time $t$ and $u(t)$ be the fed amount per time unit per fish at time $t$. The price paid per kilo of a fish of weight $x$ is taken to be of form $p(x ; t)=a_{0}+a_{1} . x$, and the cost of feed per kilo is taken to be $c$ (constant). Our objective is to determine the feed-function $u(t)$ and the time of slaughtering $T$ by maximizing the net present value,

$$
\mathrm{NPV}=x(T)\left(a_{0}+a_{1} \times(T)\right) \cdot \exp (-\rho T)-c \int_{0}^{T} u(t) \exp (-\rho T) d t
$$

subject to some growth model, which may involve weight, feeding and time $t$ (the latter may reflect both age and time of the year):

$$
\frac{d x(t)}{d t}=g(x(t), u(t), t)
$$

We have tried numerous choices for $g$, among these are

(a) $\frac{d x(t)}{d t}=-b_{0}+\frac{b_{1} u(t)}{b_{2}+u(t)}$

(b) $\frac{d x(t)}{d t}=-b_{0}+b_{1} u(t)-b_{2}(u(t))^{2}$

(c) $\frac{d x(t)}{d t}=x(t)\left(-b_{0}+\frac{b_{1} u(t)}{b_{2}+u(t)}\right)$

(d) $\frac{d x(t)}{d t}=x(t)\left(-b_{0}+b_{1} u(t)-b_{2}(u(t))^{2}\right)$

where the $b_{i}$ s are positive constants, or suitable positive functions of $t$.

In the cases $(c)$ and $(d)$ we have also considered the optimization problem for $u(t)$ being fed amount per time unit per kilo fish, and where $u(t)$ is replaced by $u(t)$. $x(t)$ in (6). We have obtained analytical solutions by control theoretic methods (the maximum principle), and studied their properties, in general, and by computational examples, see Lillestøl (1985).

Our experience may be summarized as follows: It is difficult to establish simple models for the dynamics, with solution close to observed growth and feed-functions. More realistic models will involve more parameters, but realistic values may be hard to determine or estimate from real data. Small changes in parameter values, possibly within the error limits of estimation, may give substantial change in the optimal feed-function and optimal time of slaughtering. This means that joint global modeling may lead to models which are (i) fairly complicated (ii) not easily identifiable (iii) non-robust against specification errors and (iv) not easily adaptable to changing conditions. Even if joint global models which remedy (i)-(iv) are found, we may still dismiss them because of their limited value within an interactive environment, where we want to make use of the history to date and prognosis for the immediate future.

\section{Final comments}

The theory above suffers the limitation that all fishes are supposed to be slaughtered at the same instant. For various reasons this might be an unrealistic assumption in practice. One reason may be that the fish farmer has no spot market at 
hand, and have to rely on a limited number of buyers, maybe a single one, who might ask for supply at regular or random times. Another reason may be due to the cycling of several generations within limited available space. Having specific oxygen requirements, it might be profitable to operate close to the limit which can sustain the current stock, and slaughter parts of the oldest generation when growth exceeds the sustainable limit. It is possible to formulate more general models where some density measure is included as a state variable. The theory will be more complicated, but an optimal solution may in principle be obtained using dynamic programming ideas. This is an area for further research.

In order to implement optimal solutions in practice discrete time versions of the theory may be needed. Moreover it is useful to have a framework for the updating of estimates. For these (and other) reasons one may prefer to formulate state space models and make use of Kalman filtering ideas. This is another area for further research.

This research is carried out at the Center for Applied Research (SAF) at the Norwegian School of Economics and Business Administration (NHH) with financial support from the Norwegian Fishery Research Council (NFFR).

\section{REFERENCES}

LILLeSTøL, J. (1984). Some statistical problems concerning optimal management of a fish farm. SAF-Working paper no. 11/1984 (in Norwegian).

LiLlestøL, J. (1985). Optimal management of a fish farm: Control theoretic methods applied to problems of optimal feeding and optimal slaughtering. SAF-Working paper no. $13 / 1985$ (in Norwegian). 